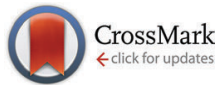

Cite this: J. Mater. Chem. B, 2016, 4, 1978

Received 22nd January 2016, Accepted 9th February 2016

DOI: $10.1039 / c 6 t b 00204 h$

www.rsc.org/MaterialsB

\title{
Bioinspired titanium coatings: self-assembly of collagen-alginate films for enhanced osseointegration $\dagger$
}

\author{
Danijela Gregurec, ${ }^{a}$ Guocheng Wang, ${ }^{\text {ab }}$ Ricardo H. Pires, ${ }^{c}$ Marija Kosutic, ${ }^{a}$ \\ Tanja Lüdtke, ${ }^{a}$ Mihaela Delcea ${ }^{c}$ and Sergio Enrique Moya*a
}

\begin{abstract}
Achieving long term osseointegration is fundamental to the development of successful bone implants. A key aspect for improving long term osseointegration on titania surfaces is to gain control of nanoand microscale features. The so called biological approach is applied here to modify the surface of titania by coating it with a self-assembled and chemically crosslinked biopolymer film made of alginate and collagen. The biofilm coated titania closely mimics the bone extracellular matrix in bio-morphology and mechanical properties. Biofilms are prepared using the layer by layer technique combined with carbodiimide chemistry to achieve a stable and compact structure. Alginate-collagen coatings display fibrillar morphology with an apparent fiber diameter of $\sim 50 \mathrm{~nm}$ and lengths ranging from a few hundred nanometers to $\sim 3 \mu \mathrm{m}$, mimicking therefore the extracellular matrix of the bone in fiber length and extent. Osteoblast MC3T3-E1 cells showed enhanced adhesion on the coated surface compared to the bare titania and a superior biological activity of the alginate-terminated coating that interfaces the cells in biological fluids.
\end{abstract}

\section{Introduction}

The optimization of surface properties of titania interfacing biological fluids and cells with respect to morphology, chemistry and bioactivity remains a major challenge in biomaterials science. Titanium and its alloys are well established materials used as implants, in particular as artificial hip joints, bone plates and dental implants. Their widespread use is attributed to their good mechanical properties, biocompatibility and high corrosion resistance. ${ }^{1,2}$ Biocompatibility of titanium mainly arises from the naturally formed surface oxide layer, $\mathrm{TiO}_{2}$, on titanium. ${ }^{3}$ However, titanium materials are often failing in long-term osseointegration processes, as they lack the ability to promote enough new bone materials for establishing a long term and secure fixation of the implant to the remnant bone. This deficiency arises primarily from the suboptimal surface properties of the implant material lacking nano- and microscale topographical features with appropriate chemical and biological characteristics. ${ }^{4}$

\footnotetext{
${ }^{a}$ Soft Matter Nanotechnology Laboratory, CIC biomaGUNE, Paseo Miramón 182 C, 20009 San Sebastian, Spain. E-mail: smoya@cicbiomagune.es

${ }^{b}$ Research Center for Human Tissues \& Organs Degeneration,

Shenzhen Institute of Advanced Technology, Chinese Academy of Science,

Shenzhen, Guangdong 518055, China

${ }^{c}$ Nanostructure Group, ZIK HIKE, Fleischmannstraße 42-44, 17489 Greifswald, Germany

$\dagger$ Electronic supplementary information (ESI) available. See DOI: 10.1039/c6tb00204h
}

In the natural bone environment, the functionality and differentiation of osteoblast cells are highly dependent on the mechanical and morphological properties of the extracellular matrix (ECM). The ECM acts as the substrate for osteoblast adhesion.

The matrix has a hybrid hierarchical mesh structure built of protein fibers, e.g. collagen and elastin, providing a scaffold support abundant with topographical information for the cells. The bone ECM consists of a large number of micro and nanotopographical features, where the collagen molecules forming collagen fibrils with diameters between $260 \mathrm{~nm}$ and $410 \mathrm{~nm}$ extend to tens of microns in length. ${ }^{5}$ The size of the apatite crystals embedded in the collagen fibrils is at the nanoscale level, measuring around 30-50 $\mathrm{nm}$ in length and 20-25 nm in width. ${ }^{6}$ In addition, a large number of nano and submicron pores are featured in the bone ECM structures providing topographical cues to the cells. Beside the chemical and topographical cues, the mechanical response, stiffness and elasticity of the matrix have a significant influence on cell activity and function. ${ }^{7}$ As cells adhere to the substrate, they closely interact with the ECM by mechanotransduction converting mechanical stimuli to biochemical signals. ${ }^{8}$ The local stiffness of the ECM that guides the cells is significantly lower than the underlying hard bone, in the range of a few $\mathrm{kPa} .{ }^{9,10}$ Therefore, a higher Young's modulus of the core biomaterial will determine its long term body implementation while low $E$ values at its surface 
will influence the activity of the cells adhered to the material prior to osseointegration.

Much effort has been devoted to developing implant architectures that closely mimic the environment of the natural bone.

The surface of implanted materials with their morphological and chemical features will provide support for cell adhesion and subsequent tissue formation leading to the fixation of the implant on the bone or it will result in an immunogenic response causing the rejection of the implant and infections of surrounding tissue. $^{4,11,12}$ Cell adhesion, migration and differentiation can be influenced by modulating the nanoscale substrate topography, i.e. by introducing nano- and microfeatures into the metallic or alloy substrate. $^{13}$

Alternatively, the so-called biological approaches can be applied, such as substrate coating with natural polymers. A biological coating introduces a biochemical environment closer to the natural one. Some examples of biological coatings are found in literature and it has been shown, for example, that a modified hyaluronan coating provides titania surfaces with enhanced release of growth factors for the activation of bone regeneration. ${ }^{14}$ Also, chitosan covalently bound to titanium alloys promotes cell proliferation and collagen fibre deposition. ${ }^{15}$

Collagen and alginate are natural polymers that are approved by the U.S. Food and Drug Administration (FDA) for human use in many types of medical applications. In the last three decades, they have been applied for wound dressings, artificial skin and tissue matrices due to their low inflammatory response and good biocompatibility. ${ }^{16,17}$

The two biopolymers are exceptional building blocks for the design of biologically inspired artificial ECMs. Collagen, a main structural protein of the connective tissue, in the form of elongated fibers found in a bone ECM, provides structural and mechanical support to the bone. ${ }^{18}$ Alginate is a natural polysaccharide known for its excellent biocompability and for having a degradation rate which depends on its molecular weight, and when incorporated in tissue scaffolds additionally can be tuned by the selection of fabrication methodology. ${ }^{19-21}$ The combination of these two polymers in a polyelectrolyte multilayer has already shown high stability and biocompatibility to endothelial cells after crosslinking with genipin, which also lead to improved cell proliferation. ${ }^{22}$

Titania is a relatively passive surface with regard to polymer adsorption if it is not functionalized with $\mathrm{OH}^{-}$groups by plasma or exposure to UV. This activation enables the electrostatically driven deposition of positively charged polymers. Alternatively, silane bound to titania, which provides charged functional groups, can be used for the electrostatic or covalent bonding of polymers. A polymer coating with collagen-hyaluronan was achieved via the so called "covalently modified" layer by layer (LbL) technique. ${ }^{23}$ The LbL technique is a simple and straightforward technique for surface functionalisation based on the alternating assembly of polyelectrolytes of opposite charge. The driving force for polyelectrolyte assembly is the electrostatic interaction between polycations and polyanions favoured by entropic considerations. The LbL assembly results in a polyelectrolyte multilayer with controlled nanoscale thickness and composition in the vertical direction.
In this study we present an approach to assemble collagenalginate multilayer films on titanium substrates forming a physiologically stable bio-inorganic hybrid interface. We will show that this interface matches the topographical and biological properties of the ECM. For the fabrication of the multilayer film we apply LbL assembly onto amino-functionalised titania combined with biopolymer cross-linking using carbodiimide chemistry. The multilayer coatings display high stability in biological fluids and a bioactivity superior to the bare titania surfaces, making them highly attractive for bone tissue engineering.

\section{Experimental}

\section{Materials}

Collagen from bovine Achilles tendon Type I, Alginic acid sodium salt, (3-aminopropyl)triethoxysilane (APTES), $N$-(3-dimethylaminopropyl)- $N$-ethylcarbodiimide hydrochloride (EDC), $N$-hydroxysuccinimide (NHS), toluene, phosphate buffered saline in tablets (PBS), sodium dodecadocyl sulfate (SDS) and Triton-X100 were all purchased from Sigma Aldrich, Madrid, Spain. Hydrochloric acid and hydrogen peroxide were obtained from Acros organic, city, country. Nanopure water water used was produced using a Diamond UV water purification system (Branstead International, IA, city, country). FITC labeled collagen and rhodamine B labeled alginate were purchased from Creative PEGWorks, Chapel Hill, USA. All the buffers, alginate and collagen solutions were filtered through the $0.2 \mu \mathrm{m}$ filters (Fisher, Madrid, Spain) and degassed in a bath sonicator prior to use. If stored, polymer solutions were kept at $4{ }^{\circ} \mathrm{C}$ in an argon atmosphere. The Falcon polystyrene tissue culture plates were purchased from Fisher Scientific (Madrid, Spain). The Actin Cytosceleton and Focal adhesion staining kit, FAK100 containing TRITC-conjugated phalloidin, anti-vinculin and $4^{\prime}$,6-diamidino-2-phenylindole (DAPI) were purchased from Merck Milipore (Madrid, Spain)

\section{Surface preparation, aminosilanisation and polymer assembly}

A semi-transparent, $25 \mathrm{~nm}$ thick $\mathrm{TiO}_{2}$ layer was coated by direct current (d.c.) magnetron reactive sputtering in an ATC $1800 \mathrm{UHV}$ sputtering system (AJA International Inc., MA) onto glass coverslips (thickness 0.13-0.16 mm, Thermo Fisher Scietific, Madrid, Spain) and a $50 \mathrm{~nm} \mathrm{Ti/TiO}$ layer was deposited onto QSX $301 \mathrm{Au}$ quartz crystals (Q-sense). Before the insertion of substrates in the magnetron, glass coverslips were cleaned for $10 \mathrm{~min}$ in a mixture of $\mathrm{H}_{2} \mathrm{O}: \mathrm{NH}_{4} \mathrm{OH}: \mathrm{H}_{2} \mathrm{O}_{2}$ (1.5:1:1 volume ratio), followed by a rinse with nanopure water, and another $10 \mathrm{~min}$ in a mixture of $\mathrm{H}_{2} \mathrm{O}: \mathrm{HCl}: \mathrm{H}_{2} \mathrm{O}_{2}\left(1.5: 1: 1\right.$ volume ratio) at $\sim 50{ }^{\circ} \mathrm{C}$, and $\mathrm{Au} \mathrm{QCM}$ crystals were cleaned in a mixture of $\mathrm{H} 2 \mathrm{O}: \mathrm{NH}_{4} \mathrm{OH}: \mathrm{H}_{2} \mathrm{O}_{2}$ (1.5:1:1 volume ratio). Clean substrates were rinsed in nanopure water and dried under nitrogen. After the insertion into the load-lock transfer chamber with a pressure of $\sim 1 \times 10^{-8} \mathrm{~Pa}$, the substrates were sputter-cleaned with a negative bias of $180 \mathrm{~V}$ $(25 \mathrm{~W})$ in a $4 \mathrm{~Pa} \mathrm{Ar}$ atmosphere for $3 \mathrm{~min}$. A 2 in. diameter Ti target (99.995\% purity, Kurt J. Lesker-USA) at a distance from the substrate of $30 \mathrm{~mm}$ with an applied d.c. power of $228 \mathrm{~W}$ at $0.4 \mathrm{~Pa}$ was used. The sputtering was performed at a room temperature 
at a substrate rotation of $80 \mathrm{rpm}$. $\mathrm{TiO}_{2}$ was deposited in the argon/oxygen atmosphere generated by combining $10 \mathrm{sccm}$ of argon flow with $20 \mathrm{sccm}$ of oxygen flow for $120 \mathrm{~min}$ and $\mathrm{Au}$ quartz crystals were sputtered for $150 \mathrm{~s}$ in an Ar atmosphere of $24 \mathrm{sccm}$ flow. Film thicknesses were calculated based on the sputtering rate measured using a built-in Quartz Crystal Microbalance (QCM) sensor in the magnetron and verified by optical ellipsometry. Surface chemical composition of the films was analyzed by X-ray photoelectron spectroscopy (XPS) after UVozone cleaning.

Aminosilanization. Surfaces were cleaned $30 \mathrm{~min}$ in $2 \%$ SDS, rinsed with nanopure water, dried under nitrogen stream and treated in an UV-ozone chamber for $30 \mathrm{~min}$. Surfaces were immediately inserted into a 1\% solution of APTES in toluene. Aminosilanisation was carried out overnight at $115{ }^{\circ} \mathrm{C}$, the temperature of boiling toluene. In order to remove excess of APTES, surfaces were cleaned three times in fresh toluene followed by washing three times in nanopure water with sonication and each cleaning cycle lasting $30 \mathrm{~min}$.

Polymer deposition. A schematic representation of biofilm assembly is shown in Fig. 1a. Polymer films were assembled onto aminosilanised titania surfaces by alternately dipping into polymer solutions with $0.5 \mathrm{mg} \mathrm{mL} \mathrm{mL}^{-1}$ polymer concentration starting with alginate dissolved in PBS followed by collagen and alginate in $\mathrm{Na}$ acetic buffer at $\mathrm{pH} 4$ up to a desired number of layers. For the crosslinking, samples were shortly rinsed in PBS buffer at a pH 7.4 and immersed into EDC/NHS dissolved in PBS buffer at a 6.5:1 mass ratio. After the crosslinking reaction, the samples were rinsed with PBS to remove excess of crosslinker. Crosslinking takes place through the formation of an amide bond between $\mathrm{COOH}$ and $\mathrm{NH}_{2}$ groups as shown in Fig. $1 \mathrm{~b}$.

\section{Quartz crystal microbalance with dissipation monitoring} (QCM-D)

The assembly of collagen-alginate films was monitored using an E4 QCM-D from Q-Sense, Goteborg, Sweden. QSX 301 Au quartz crystals (Q-sense) were coated with $\mathrm{Ti} / \mathrm{TiO}_{2}(50 \mathrm{~nm})$, and then functionalised with APTES prior to assembly in the QCM-D chamber.

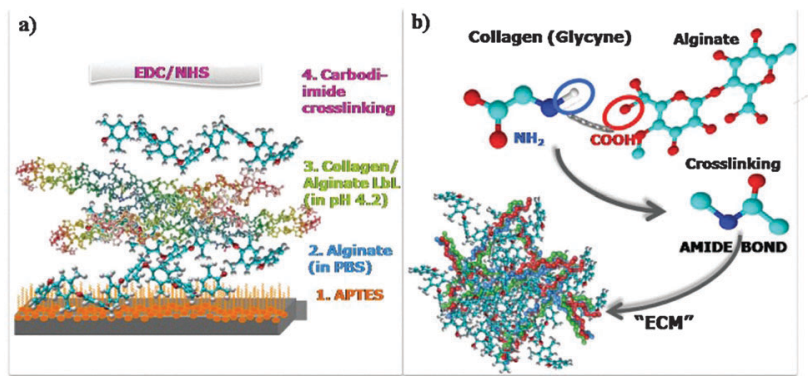

Fig. 1 Schematic representation of the alginate collagen film assembly (a) and film crosslinking (b) to produce an "ECM-like" structure. In (a) $\mathrm{TiO}_{2}$ is first functionalised with APTES and alginate is deposited as the first layer in PBS. Assembly of collagen/alginate following layers is performed in acidic medium up to a desired layer number and film is finally crosslinked according to scheme in (b); amino groups of glycine are covalently linked to carboxyl groups of alginate forming amide bonds.
The LbL assembly was performed by passing $0.5 \mathrm{mg} \mathrm{mL}^{-1}$ polyelectrolyte solution in $0.1 \mathrm{M}$ Na acetate buffer $(\mathrm{pH} 4)$ with a peristaltic pump through the chamber. LbL deposition was monitored by the decrease in the resonance frequency of the quartz crystal. Once the frequency values stabilized, the polymer solution was replaced by a Na acetate buffer until a plateau in the frequency response was recorded. This procedure was repeated for the deposition of a desired number of polyelectrolyte layers, alternating collagen and alginate. After deposition, the film was shortly rinsed with PBS buffer to exchange $\mathrm{pH}$ and further crosslinked by passing EDC/NHS $\left(6.5 / 1 \mathrm{mg} \mathrm{mL}^{-1}\right)$ in PBS through the chamber.

\section{X-ray photoelectron spectroscopy (XPS)}

Surface analysis by XPS was performed in a SPECS SAGE HR 100 system spectrometer. The X-ray source employed for this analysis was $\mathrm{Mg} \mathrm{K} \alpha(1253.6 \mathrm{eV})$ which operated at $12.5 \mathrm{kV}$ and $250 \mathrm{~W}$. The take-off angle was fixed at $90^{\circ}$ and the analysis was conducted at a pressure of $\sim 10^{-6} \mathrm{~Pa}$. Surfaces were brought into the XPS chamber within $5 \mathrm{~min}$ after cleaning/preparation. Survey spectra were obtained with a pass energy of $30 \mathrm{eV}$ and detailed spectra were acquired for $\mathrm{C} 1 \mathrm{~s}, \mathrm{O} 1 \mathrm{~s}$, Ti $2 \mathrm{p}$ and $\mathrm{N} 1 \mathrm{~s}$ regions with a pass energy of $15 \mathrm{eV}$. Spectra were analyzed using CasaXPS 2.3.15dev87 software. The analysis consisted of satellite removal, Shirley background subtraction, calibration of the binding energies related to the C 1s C-C peak at $285 \mathrm{eV}$, and peak fitting with Gaussian-Lorentizan line shapes to determine the atomic percentages and chemical states of elements present on the surface.

\section{Atomic force microscopy (AFM)}

Structural details of the collagen-alginate films were investigated using a Nanowizard II AFM (JPK, Berlin, Germany) and a Nanoscope V Multimode AFM (Santa Barbara, CA). Tapping mode imaging was performed in liquid (PBS or Na acetate buffers) using a DNP-S10A cantilevers (Bruker, Berlin, Germany) with a nominal spring constant $0.350 \mathrm{~N} \mathrm{~m}^{-1}$ and resonant frequency at $\sim 13 \mathrm{kHz}$. In order to measure the height of the films, nanolithography was performed on a Nanowizard II AFM with TESP-V2 cantilever (Bruker, Berlin, Germany) with a nominal spring constant of $40 \mathrm{~N} \mathrm{~m}^{-1}$ and the resonant frequency was $\sim 130 \mathrm{kHz}$. Lithography was achieved by defining an area of $8 \mu \mathrm{m} \times 2 \mu \mathrm{m}$ that was rasterized in contact mode at $160 \mu \mathrm{m} \mathrm{s}^{-1}$ and subsequently imaged. This was repeated several times, until a smooth surface of the substrate could be observed and no increment in the height of the film edges could be seen. After performing nanolithography, tip direction was rotated for $90^{\circ}$ to acquire images and measure height profiles.

\section{Confocal laser scanning microscopy (CLSM) and biological experiments}

Fluorescence imaging of labelled polymers and cells was performed using a Zeiss LSM 510 confocal microscope (Carl Zeiss, Göttingen, Gremany), MC3T3-E1 cells were used to evaluate the biocompatibility and bioactivity of the produced films. Cell culture and cell seeding on the films were performed as previously described. ${ }^{13}$ Briefly, cells were cultured with $\alpha$-MEM 
(Life technologies, Madrid, Spain) supplemented with $10 \%$ fetal bovine serum and 1\% penicillin/streptomycin (both from Sigma Aldrich, Madrid, Spain). Cells were cultured at $37{ }^{\circ} \mathrm{C}$ in an atmosphere of $5 \% \mathrm{CO}_{2}$ and the medium was refreshed every three days. When the cells reached $80 \%$ confluence, they were trypsinized and resuspended in fresh medium to arrive at a cell suspension with a final cell density of $3 \times 10^{4} .1 \mathrm{~mL}$ of cell suspension was added into each well of 12 well cell culture plates for adhesion experiments and in 24 well plates for determination of proliferation. The titanium samples had been placed in the plate wells and sterilized by UV irradiation for 60 minutes prior to cell addition. After culturing for 24 hours, cells were fixed in a $4 \%$ paraformaldehyde solution. F-actin, nuclei and focal adhesion of the cells were stained using the actin cytoskeleton and focal adhesion staining kit FAK100 (Merck Millipore, Madrid, Spain). Briefly, cells cultured on the surface of the films were first permeabilized with TritonX100 (Sigma) for $4 \mathrm{~min}$ at room temperature. Then, the cells were incubated in dilute primary antibody (anti-vinculin) solution for 1 hour at room temperature, followed by washing three times with PBS for 5-10 min each, and then incubated with a secondary antibody (FITC-conjugated) solution and TRITC-conjugated Phalloidin for 1 hour at room temperature. After three washing steps with PBS, cells were incubated with DAPI for $3 \mathrm{~min}$ at room temperature, followed again by three washing steps with PBS. A cell proliferation colorimetric assay was conducted using the Cell Counting Kit-8 (CCK-8) (Sigma Aldrich, Madrid, Spain) containing [2-(2-methoxy-4-nitrophenyl)3-(4-nitrophenyl)-5-(2,4-disulfophenyl)-2H-tetrazolium, monosodium salt], (WST-8), a nontoxic dye used for continuous cell culturing. Colorimetric analysis was performed after 3 and 7 days of cell culturing using a plate reader (GENios Pro, Tecan) equipped with a $450 \mathrm{~nm}$ filter.

For alkaline phosphatase (ALP) staining, cells were seeded with a density of $2 \times 10^{4}$ cells per mL. Cells were differentiated in osteogenic medium that is additionally supplemented with a $50 \mu \mathrm{g} \mathrm{mL}^{-1}$ of ascorbic acid and $2 \mathrm{mM} \beta$-betaglycerophosphate.

After culturing for 12 days in osteogenic medium, cells were fixed with a $4 \%$ paraformaldehyde solution, and then, stained using an ALP staining kit (1-Step TM NBT/BCIP, Thermo) for $1 \mathrm{~h}$. The ALP area is determined using the MacBiophotonics ImageJ program and further statistical analysis concerning ANOVA analysis is done using OriginPro 2016 software.

\section{Results and discussion}

\section{Biopolymer film fabrication}

Multilayer biopolymer films of alginate and collagen were prepared on titania substrates by means of the layer by layer (LbL) deposition in acid medium where collagen has a positive charge and alginate maintains negative charge. After multilayer assembly, the film was quickly rinsed with PBS to exchange the acidic medium under physiological conditions and films were finally crosslinked via carbodiimide chemistry. A schematic representation of the procedure followed for film fabrication is shown in Fig. 1a. In order to enable stable bonding between the $\mathrm{TiO}_{2}$ surface and the biopolymer film, surfaces were aminosilanized prior to film deposition with APTES providing the surface with $\mathrm{NH}_{2}$ groups, which are easily bound to the $\mathrm{COOH}$ groups of alginate using carbodiimide chemistry. Successful silanization of titania was confirmed by X-ray photoelectron spectroscopy (XPS) as revealed in Fig. S1 (ESI $\dagger$ ). In this figure we show a survey surface chemistry spectra of bare $\mathrm{TiO}_{2}$ (in black) and $\mathrm{TiO}_{2}$ functionalised with APTES (in red). The inset in Fig. S1 (ESI $\dagger$ ) shows the appearance of a peak at $401 \mathrm{eV}$, assigned to the $\mathrm{NH}_{2}$ group in the nitrogen 1s region for APTES functionalized $\mathrm{TiO}_{2} \cdot{ }^{24}$ The assembly of the biofilm performed without silanization of the $\mathrm{TiO}_{2}$ surface was not stabile in cell culture medium and detached from the substrate (data not shown). This is understandable considering the relatively smooth titania surface used here, which results in a low surface charge density and therefore, does not provide enough $\mathrm{OH}^{-}$ groups for strong electrostatic bonding of the polymers. ${ }^{25,26}$

The average zeta potential ( $\mathrm{zP}$ ) of alginate is more negative in PBS $(-30.0 \pm 1.5 \mathrm{mV})$ than in acetic buffer at a pH $4(-7.8 \pm$ $4.4 \mathrm{mV}$ ). This trend for the $\mathrm{zP}$ of alginate with increasing $\mathrm{pH}$ is in agreement with a study of Carneiro-da-Cunha et al. where authors investigated the influence of $\mathrm{pH}$ on $\mathrm{zP}$ of polysaccharide solutions ${ }^{27}$ and it can be expected that the higher the $\mathrm{pH}$ the more ionized the carboxilic groups are. Therefore, the first alginate layer was deposited in PBS buffer followed by the alternating deposition of collagen and alginate in $\mathrm{Na}$ acetic buffer at a pH 4 where collagen is dissolved and has a positive charge. A limitation for the deposition under physiological conditions arises partially from collagen structure; at the isoelectric point, around physiological 7.4, amino acids across the collagen molecule have a net charge close to zero. This results in the aggregation of the collagen molecules, which precipitate becoming insoluble. At acidic $\mathrm{pH}$, on the other hand, the collagen molecules are positively charged and collagen can be dissolved in the medium. Therefore, the whole assembly was first performed under acidic conditions. To achieve a compact structure and physiologically stable films on titania, we performed the crosslinking of the carboxylic groups of alginate and the amino groups of proline, accessing amino acid of the collagen molecules via cabodiimide chemistry using EDC/NHS as shown in the scheme in Fig. 1b. Crosslinking resulted in the formation of an amide bond between the two polymers. Prior to crosslinking, films were shortly rinsed with PBS to increase the $\mathrm{pH}$ as the NHS ester-activated cross-linker reacts with primary amines under physiological to slightly alkaline conditions. The crosslinking and the changes to physiological $\mathrm{pH}$ will result in a fibrillar network structure arising from collagen fibres. Changing the $\mathrm{pH}$ from acid to physiological causes the precipitation of the collagen proteins that will then form fibres as the nucleation of the collagen molecules is $\mathrm{pH}$ induced and growth into fibres occurs around $\mathrm{pH} 7$ at a concentration of $0.5 \mathrm{mg} \mathrm{mL}^{-1}$ used for assembly of these films. ${ }^{28}$

The in situ assembly of the film on an aminosilanized titania surface was followed by means of the quartz crystal microbalance using the dissipation monitoring (QCM-D) technique as shown 

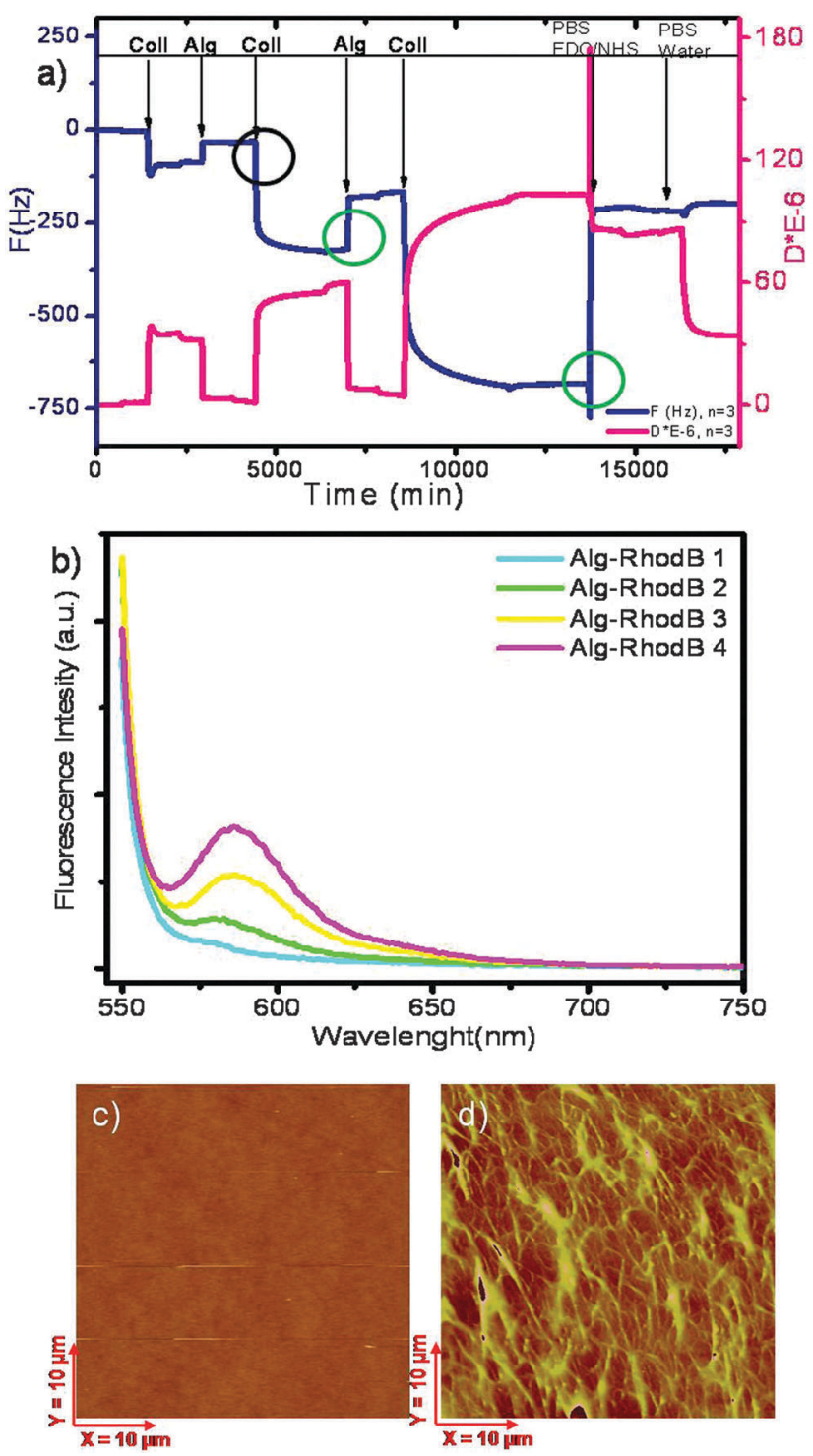

Fig. 2 Biofilm assembly and characterization. (a) Alginate-collagen assembly followed by QCM-D, green circles represent a frequency increase after alginate deposition and upon change to PBS and crosslinking at the end, black circles represent the change in frequency upon deposition of collagen. (b) Fluorescence intensity curves for films including rhodamine B-labelled alginate. (c) Nanoscope AFM image of the bare titania surface and (d) Nanoscope AFM image of a 9 layer collagenalginate film deposited on top of a silanized surface.

in Fig. 2a. The film assembly was followed at the 3rd overtone, referred to in figure as " $n=3$ ". In QCM-D experiments the frequency $(\Delta F)$ of the crystal decreases proportionally to the mass deposition. ${ }^{29}$ In the experiment shown in the figure, we used a titania covered quartz crystal sensor. The coating with titania is described in the experimental part. Prior to the QCM-D measurements, crystals were silanized outside of the device. Silanization was not performed in the QCM-D chamber because the toluene solvent used in the process is not compatible with the QCM-D device. In the QCM-D chamber we deposited a film of up to 9 layers observing a decrease in frequency after each collagen deposition ranging between 220 to $310 \mathrm{~Hz}$ as noted in the black circle in Fig. 2a, but also a relatively high frequency increase of $\sim 120 \mathrm{~Hz}$ after every deposition of alginate, as indicated in the figure by a green circle on the left. Frequency also largely increases, $\sim 400 \mathrm{~Hz}$, upon changing the $\mathrm{pH}$ to 7.4 followed by crosslinking with EDC/NHS in PBS as shown in the green circle on the right in the graph. Similarly, increase in frequency upon alginate deposition was also observed in the study of Chaubaroux et al. where they followed collagen-alginate assembly on $\mathrm{SiO}_{2}$ crystals. ${ }^{22}$ The frequency increase observed by QCM-D can be indicative of polymer removal but it can also be related to the removal of water in the film and changes in viscoelastic properties of the film associated with the water liberation. A decrease in the water content in the film, which would result in a decrease in frequency, implies a densification of the layers and a more rigid and stable structure. Despite the increase in frequency for alginate, the film assembles as the total frequency continues to decrease with increasing number of layers.

In order to gain more information on the alginate assembly, we assembled rhodamine $B$ labeled alginate and measured the fluorescence emission spectra after each alginate deposition. In Fig. 2b we show the fluorescence spectra of the collagenalginate film assembled on the $\mathrm{TiO}_{2}$ surface for four deposition processes of alginate. Fluorescence intensity clearly increases with increasing number of labelled layers. Moreover, the increase of the rhodamine B fluorescence is linear as shown in Fig. S2 (ESI $\dagger$ ). The fluorescence data here serve as evidence that alginate deposits at each deposition step.

\section{Morphological and mechanical characterization of the biofilms}

Furthermore, AFM imaging reveals a fibrillar structure arising from collagen fibres after the crosslinking as seen from the topography image in Fig. 2c. The height of the film (shown in Fig. S3a, ESI $\dagger$ ) was $30 \mathrm{~nm}$ for 9 layer film assembly. To measure the height, we removed an area of the film revealing the substrate, as explained in the Methods section and determined a $z$ profile of the film from the step separating the substrate and film deposited on the substrate. The diameters of fibres ranged from $\sim 20$ to $\sim 120 \mathrm{~nm}$ with most of them having a diameter of $\sim 50 \mathrm{~nm}(49.0 \pm 27 \mathrm{~nm})$ as calculated for the average value. The length of long fibres is in the micrometer range, from a couple of hundred nanometres to $\sim 3 \mu \mathrm{m}$ and the distance between two parallel fibres vary from $\sim 100 \mathrm{~nm}$ to $\sim 600 \mathrm{~nm}$. The length can be seen more clearly on a phase image of a smaller scale, shown in Fig. S3b (ESI $\dagger$ ). The fibrillar network presented in this work reassembles the topology of the natural ECM, which is reported to be formed by fibres with roughly $50 \mathrm{~nm}$ in diameter and with lengths of several micrometers. ${ }^{30}$ The coating based on our strategy gives more compatibility to the implant material as we will show later in the manuscript. Prior to access the biocompatibility and bioactivity tests, structural changes upon pH switch and crosslinking were investigated by AFM. In Fig. 3a we show a 5 layer film assembled in acid before $\mathrm{pH}$ changes and crosslinking. This assembly has some network like structure but during imaging we could notice a "wobbly" structure where the features of the film are drifting with the tip during scanning even 

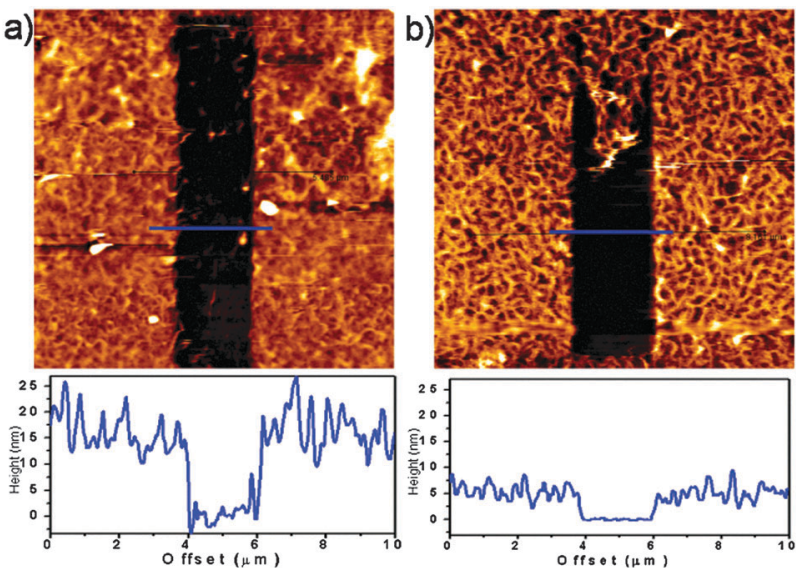

Fig. 3 AFM topography and height profile. $10 \times 10 \mu \mathrm{m}$ performed by JPK Topography of 5 layer films prepared as (a) LbL, in acid medium and (b) after crosslinking imaged in PBS. Bottom panels are height profiles measured at a cross section indicated in figures (a) and (b).

by use of very soft tip, implying a film of limited stability. However, the height of this film before crosslinking is three times larger than that after crosslinking. A thickness of approx. $15 \mathrm{~nm}$ was measured for the LbL assembled film and of $\sim 5 \mathrm{~nm}$ for the crosslinked one, as shown in Fig. 3, bottom panels. After crosslinking, we observed a more pronounced fibrillar structure (Fig. 3b) and no drifts of film features during imaging. We can conclude that crosslinking induces a more stable and compact film, developing the fibrillar structure. Film mechanics was further investigated by nanoindentation and by measuring the Young's elastic modulus that allows us to correlate elastic properties to changes in the structural and morphological properties of the films. The Young's modulus (modulus of elasticity, E) was determined using Atomic Force Spectroscopy (AFS) measuring the force applied by the tip of a cantilever as a function of its indentation into the sample resulting in force distance $(F-d)$ curves. The results from nanoindentation experiments are presented in the ESI $\dagger$ with detailed information on how experiments were performed. Apparent $E$ values of $\sim 35 \mathrm{kPa}$ for LbL deposited films and $\sim 10 \mathrm{kPa}$ for crosslinked with a higher error for non-crosslinked (LbL) films were measured. Though the $E$ values themselves are in a nice agreement with the literature for collagen fibers ${ }^{31}$ as well with the elasticity of soft bone tissue of $25-40 \mathrm{kPa}^{10}$ we have also concluded that the values of $E$ are highly affected by the substrate and as such can only be considered as apparent. It can also be concluded from indentation measurements that the film is highly homogeneous. This is manifested in the standard deviation of the mean $E$ value (distribution curves in Fig. S5c and S5d, ESI $\dagger$ ). Wider distribution of apparent Young's modulus in the case of the LbL films indicates less homogeneous coating due to variability in the indentation curves across the sample while crosslinked films seems to be more homogeneous regarding their elastic properties.

\section{Biocompatibility and bioactivity of the biopolymer films}

Once the stable crosslinked films on titania were prepared and characterized, their biocompatibility was evaluated by investigating the influence of the films on cell adhesion and proliferation. Additionally, we have studied the impact of the coatings on cells measuring the cellular ability to deposit mineralised tissue. Biological evaluation reveals important information on how the osteoblast cells interact with the biopolymers. First, we used CLSM to visualize the structure of the films assembled by rhodamine B labelled alginate and FITC labelled collagen films. As seen in Fig. 4a, collagen shows a pronounced fibrillar structure, while alginate is homogeneously distributed over the film. In Fig. 4c we merged the rhodamine B channel with the transmission light channel used to visualize pre-osteoblast cells, and we noticed that the areas of high intensity of rhodamine labelled alginate overlays in space with the cell nuclei. This is, however, not the case with collagen for which we did not observe the cells following the path of the collagen fibers. Also, comparing films with alginate and collagen as the top layer we observed differences implying that cells grow better when alginate is deposited as a last layer as shown in Fig. 4b where we see more cells with apparently larger dimensions than in the case of the coating where collagen is deposited as a last layer (Fig. 4a). Results of the proliferation tests are shown in Fig. 5. There is no significant difference in the proliferation rate of the cells cultured on bare titania, with the film with collagen as the last layer and
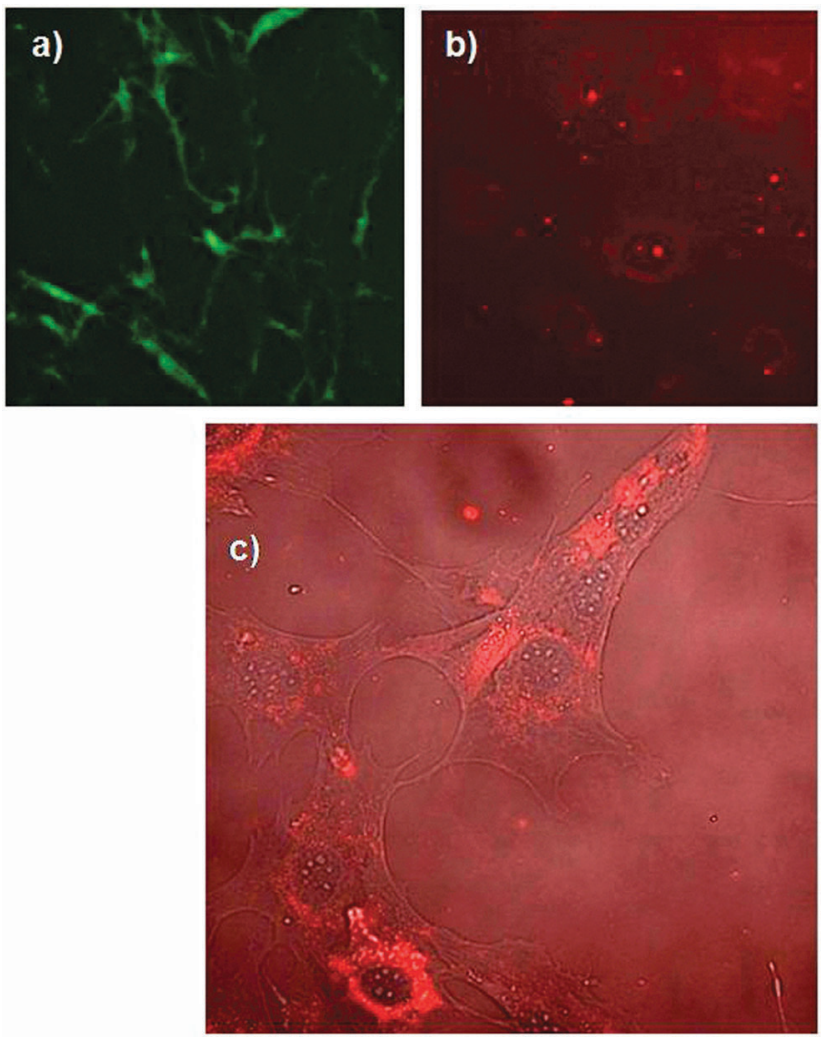

Fig. 4 3D structure of films by CLSM (a) 4 layer film prepared with collagen-FITC and non-labeled alginate, collagen is deposited as the last layer and (b) 5 layer film prepared from non-labeled collagen and alginatelabeled with rhodamine B, with alginate being the last deposited layer. (c) Merged transmission light channel with the rhodamine-B channel for pre-osteoblast cells incubated 24 hours. $40 \times$ magnification images have a size of $225 \times 225$ micrometers. 


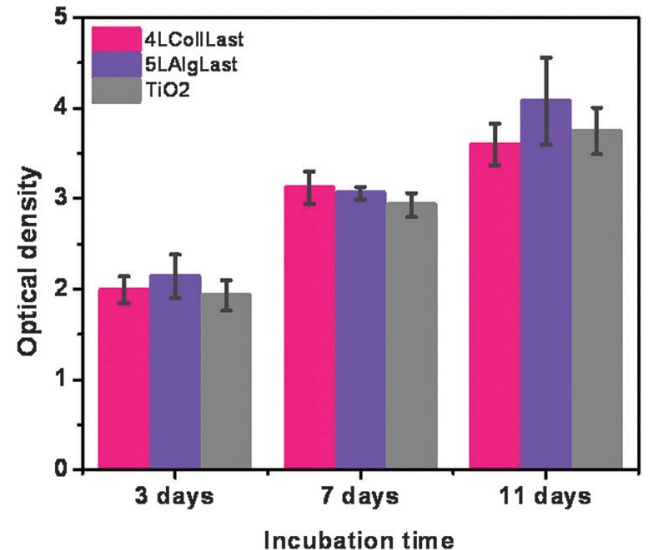

Fig. 5 Proliferation of pre-osteoblast cells cultured on crosslinked films of 4 layer collagen-alginate film with collagen deposited as a last layer (pink), 5 layer film with alginate deposited as a last layer (purple) and bare $\mathrm{TiO}_{2}$ serving as a control (gray).

films with alginate as the last layer, indicating that the biocompatibility of the assembled films is comparable to the bare titania film. From this result, we can conclude that the coated films promote proliferation of pre-osteoblast cells as well as titania do. The biopolymer coating is expected to have however an impact on cell functions, especially on cellular adhesion. The initial adhesion of cells on biomaterials is a key regulator of cell proliferation, migration and differentiation, which lately determines the fate of the biomaterial. Pre-osteoblast cells were cultured on titania coated with collagen-alginate 6 layers film with collagen as the top layer (Fig. 6a) and collagen-alginate 5 layer film with alginate as the top layer (Fig. 6b) to evaluate the individual effects of collagen and alginate upon adhesion of pre-osteoblasts. Cells were cultured on bare titania as a control sample shown in Fig. 6c. Cells staining of vinculin (green),

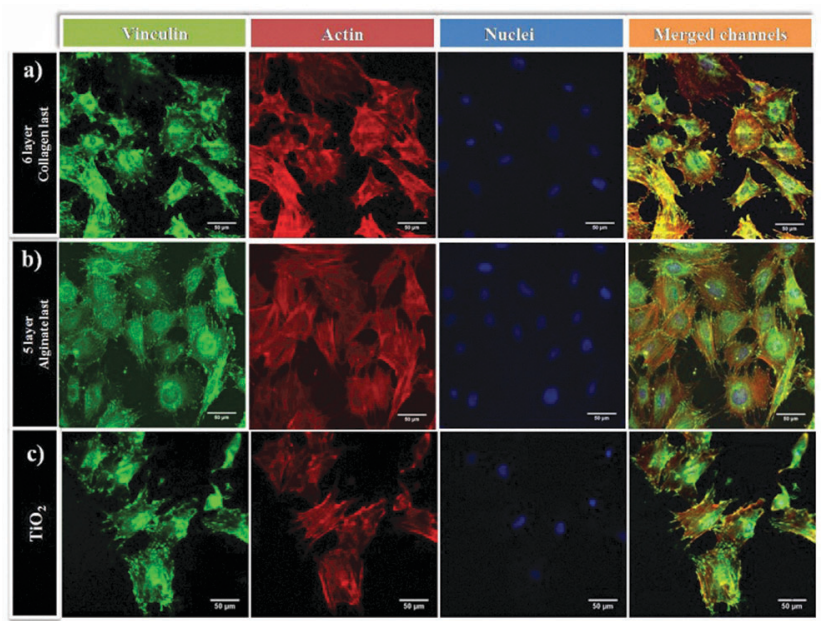

Fig. 6 CLSM images of focal adhesion and nucleus staining after 24 hours on the crosslinked films (a) 6 layer film with the collagen last layer, (b) 5 layer film with the alginate last layer and (c) the bare titania surface. The green channel is vinculin staining, red actin, blue nucleus and the last panel are all channels merged into one.
F-actin (red) and nucleus (blue) are shown in the first, second and third panels, respectively, and merged channels are shown in the last panel. On average, cells cultured on bare titania are smaller and less numerous than those cultured on alginateterminated films. They also show a diffuse cytoskeleton with less stress fibres in their cytoplasm as observed in the images of F-actin staining. In the images showing vinculin staining, more green spots can be seen at the border of the cells cultured on the film with alginate as a last layer compared to the cells cultured on film with collagen as a last layer. We can also distinguish more peri-nucleus regions displaying a bright green colour in the cells cultured on the film finishing with alginate. From the images of F-actin, in the red channel, we appreciate that cells cultured on the films with last layer alginate are somewhat larger in size and show a rearranged cytoskeleton with distinctive stress fibres inside the cytoplasm. On average, the cell number on the multilayer coated titania is higher than that on bare titania, as shown in Fig. S6 (ESI $\dagger$ ), where we can see that there are $50 \%$ more cells on the multilayer terminated with alginate in comparison with the cell number on titania. These results indicate enhanced focal adhesion first on the coated titania compared to bare titania, and a better focal adhesion when alginate is the last layer, corresponding to the observation of the presence of alginate around cell nuclei discussed above, Fig. 4b. One would expect that alginate decreases cell adherence as it is known to have antifouling properties and limited interaction with cells. However, it is necessary to bear in mind that the properties of layer by layer films are a combination of those of both the cationic and anionic polymers. In the particular case of using fibers like collagen and a lineal polymer like alginate the assembly of the alginate at each layer deposition is restricted to the collagen fibers, which do not form a homogeneous structure. Because of geometrical consideration each deposition of collagen will result in a different arrangement of fibers and each layer of alginate will deposit on the surface of the fibers. Under these conditions there will only be one layer of alginate on top of each collagen layer and consequently the alginate will not form a dense coating on the collagen fibers. If the alginate is not covering the fibers homogeneously we can expect that the antifouling action be limited. Most likely cells will sense the collagen as well, which will probably contribute to cell adherence. The improved cell adherence with alginate as the top layer over the adherence with collagen as the top layer must be related to the alginate uptake by the cells.

Finally, we evaluated the alkaline phosphatase (ALP) activity of the differentiating osteoblast cells on the crosslinked films and titania. ALP activity is used as a gene marker in the early stage of osteogenic differentiation and to determine the ability of the cells to deposit mineralized tissue. Optical images of ALP stained osteoblasts after culturing in osteogenic medium for 12 days are shown in Fig. $7 \mathrm{a}-\mathrm{c}$, for alginate terminated film, collagen terminated film and bare titania as a control sample respectively. Cells cultured on the coated titania after staining exhibit a more of darker blue-purple color, compared to cells cultured on $\mathrm{TiO}_{2}$. These results indicate that the cells cultured 

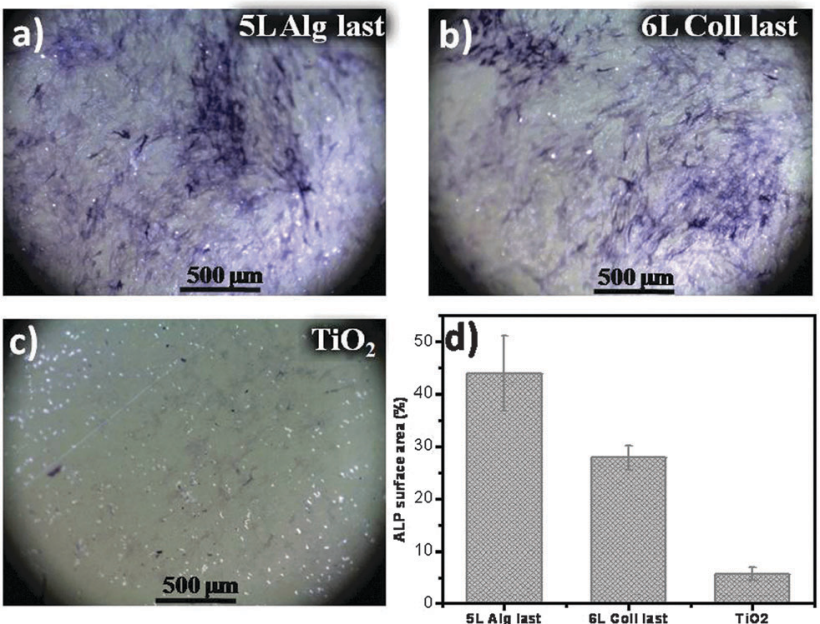

Fig. 7 ALP activity of osteoblast cells after 12 days of mineralization. Images of ALP staining for osteoblast 12 days cultured on (a) 5 layer alginate terminated coating, (b) 6 layers coated titania terminated with collagen and in (c) the $\mathrm{TiO}_{2}$ control sample. In (d) ALP staining of osteoblasts, expressed as the ALP stained surface area.

on the multilayer coated titania have enhanced ALP activity implying the positive effects of biopolymers on the osteoblast differentiation and mineralization ability. The staining was quantified by measuring the ALP positive area (Fig. 7d). Area quantification shows that ALP activity on samples with alginate as the top layer is 9 times larger than on bare titania while on collagen terminated films this increase is 6 times larger than titania. Our results show a positive effect of the films on mineralization and in agreement with the adhesion experiments show a better performance when alginate is the top layer. Statistical analysis of mean values shows a significant difference between both coated titania surfaces compared to bare titania by Tukey analysis. Comparison of significant differences in mean values is shown in Fig. S6 (ESI†).

The observation that alginate results in a better cell functionality correlates with the uptake of alginate, which is not observed when collagen is the top layer. Alginate, an anionic polysaccharide, could serve as an energy source for the cells when degraded to monosaccharides. ${ }^{32}$ Alginate degradation is slow under physiological conditions but since we assemble alginate at $\mathrm{pH} 4$ the molecule may be partially hydrolyzed when is taken up by the cells. ${ }^{33}$ There may also be a specific effect of alginate on the functionality of osteoblasts, which has not been reported before. Further biological studies should be conducted to understand the relationship between the improved functionality of osteoblasts and alginate uptake.

\section{Conclusions}

We reported the fabrication of highly biocompatible coatings on aminosilanised titania substrates via layer by layer assembly of the natural polyelectrolytes, collagen and alginate. LbL assembly was conducted at low $\mathrm{pH}$ followed by a reversal of $\mathrm{pH}$ to more basic media and crosslinking by carbodidimide chemistry.
A compact fibrilar structure is produced that morphologically and chemically mimics bone's extracellular matrix. This fibrilar structure can be used to enhance the biocompatibility of currently used titania implant materials. Stability and morphology characterisation of the coatings revealed highly stable coatings. We found that these coatings enhanced the adhesion of osteoblast cells compared with the bare titania surfaces, with a superior cell activity of the alginate-terminated films. Ultimately, we have presented a strategy for the assembly of biocompatible and stable coatings based on two natural molecules on top of the clinically relevant titania substrate mimicking the ECM structure. The coated surfaces display more significant bioactive properties than bare titania and present high potential for overcoming body implant rejections.

\section{Acknowledgements}

The authors acknowledge the project MAT2013-48169-R from the Spanish Ministry of Economy (MINECO) and the German Ministry of Education and Research (BMBF) within the project FKZ 03Z2CN11. G. C. Wang thanks the Shenzhen Peacock Innovation Team (110811003586331) and Shenzhen Science and Technology Research funding (ZDSY20130401165820356, JCYJ20150630114942256) for their support.

\section{References}

1 M. B. Nasab, M. R. Hassan and B. B. S. Sahari, Trends Biomater. Artif. Organs, 2010, 24, 69-82.

2 M. Geetha, A. K. Singh, R. Asokamani and A. K. Gogia, Prog. Mater. Sci., 2009, 54, 397-425.

3 B. Kasemo, J. Prosthet. Dent., 1983, 49, 832-837.

4 G. Wang, S. E. Moya, Z. Lu, D. Gregurec and H. Zreiqat, Nanomedicine, 2015, 10, 1327-1341.

5 C. J. Bettinger, R. Langer and J. T. Borenstein, Angew. Chem., Int. Ed., 2009, 48, 5406-5415.

6 L. C. Palmer, C. J. Newcomb, S. R. Kaltz, E. D. Spoerke and S. I. Stupp, Chem. Rev., 2008, 108, 4754-4783.

7 D. W. DeSimone, R. P. Mecham, Y. Choi, A. Holle and A. Engler, Development, Biology of Extracellular Matrix, Springer, Springer-Verlag, Berlin, Heidelberg, 2013.

8 S. K. Bhatia, B. Mason, J. Califano and C. Reinhart-King, Engineering Biomaterials for Regenerative Medicine, Springer, New York, 2011.

9 Y. Zhu, Z. Dong, U. C. Wejinya, S. Jin and K. Ye, J. Biomech., 2011, 44, 2356-2361.

10 H. Lv, L. Li, S. Meiyu, Y. Zhang, L. Chen, Y. Rong and Y. Li, Stem Cell Res. Ther., 2015, 6, 103.

11 D. Li, J. Zhou, F. Chowdhury, J. Cheng, N. Wang and F. Wang, Regener. Med., 2012, 6, 229-240.

12 F. M. Watt and W. T. S. Huck, Nat. Rev. Mol. Cell Biol., 2013, 14, 467-473.

13 L. Yate, L. E. Coy, D. Gregurec, W. Aperador, S. E. Moya and G. Wang, ACS Appl. Mater. Interfaces, 2015, 7, 6351-6358.

14 I. Berts, D. Ossipov, G. Fragneto, G. A. Frisk and A. R. Rennie, Adv. Eng. Mater., 2014, 16, 1340-1350. 
15 H. Y. Lin and J. H. Chen, Carbohydr. Polym., 2013, 97, 618-626.

16 J. F. Cavallaro, P. D. Kemp and K. H. Kraus, Biotechnol. Bioeng., 1994, 43, 781-791.

17 J. A. Rowley, G. Madlambayan and D. J. Mooney, Biomaterials, 1999, 20, 45-53.

18 L. D. Muiznieks and F. W. Keeley, BBA, Mol. Basis Dis., 2013, 1832, 866-875.

19 H. Park, K. Y. Lee, R. L. Reis, N. M. Neves, J. F. Mano, M. E. Gomes, A. P. Marques and H. S. Azevedo, Natural-Based Polymers for Biomedical Applications, Woodhead Publishing, 2008, pp. 515-532.

20 S. Jinchen and T. Huaping, Materials, 2013, 6, 1285-1309.

21 H. J. Kong, D. Kaigler, K. Kim and D. J. Mooney, Biomacromolecules, 2004, 5, 1720-1727.

22 C. Chaubaroux, E. Vrana, C. Debry, P. Schaaf, B. Senger, J. C. Voegel, Y. Haikel, C. Ringwald, J. Hemmerle, P. Lavalle and F. Boulmedais, Biomacromolecules, 2012, 13, 2128-2135.

23 H. Ao, Y. Xie, H. Tan, S. Yang, K. Li, X. Wu, X. Zheng and T. Tang, J. R. Soc., Interface, 2013, 10, 20130070.
24 G. Beamson and D. Briggs, In High Resolution XPS of Organic Polymers - The Scienta ESCA300 Database, John Wiley and Sons, Chichester, 1992.

25 U. Diebold, Surf. Sci. Rep., 2003, 48, 53-229.

26 F. Borghi, V. Vyas, A. Podesta and P. Milani, PLoS One, 2013, 8, e68655.

27 M. G. Carneiro-da-Cunha, M. A. Cerqueira, B. W. S. Souza, J. A. Teixeira and A. A. Vicente, Carbohydr. Polym., 2011, 8, 522-528.

28 G. C. Wood, Biochem. J., 1960, 75, 598-605.

29 K. Kanazawa and N.-J. Cho, J. Sens., 2009, 2009, 1-17.

30 H. Lodish, A. Berk, S. L. Zipursky, P. Matsudaira, D. Baltimore and J. Darnell, Molecular Cell Biology, W. H. Freeman, New York, 4th edn, 2000.

31 Y. Zhu, Z. Dong, U. C. Wejinya, S. Jin and K. Ye, J. Biomech., 2011, 44, 2356-2361.

32 D. M. Hillis, D. E. Sadava, H. C. Heller and M. V. Price, Principles of Life, Macmillan Higher Education, London, 2011.

33 S. Holtan, Q. Zhang, W. I. Strand and G. Skjak-Braek, Biomacromolecules, 2006, 7, 2108-2121. 